\title{
Verification of Overlapping Effects of Proprioceptive Training and Electrical Stimulation Therapy on Reducing Upper Limb Spasticity and Improving Physical Functions and Activities of Daily Living
}

\author{
Jung-Ho Lee ${ }^{1}$, Youn-Bum Sung ${ }^{2}$ and Dae-Hwan Lee ${ }^{3 *}$ \\ ${ }^{1}$ Dept. Physical Therapy, Kyungdong Univ., Bongpo-ri, Toseong-myeon, Goseong- \\ gun, 219-705, Gangwon-do, Republic of Korea \\ ${ }^{2}$ Dept. of Physical Therapy, Daegu University, 201, Naeri-ri, Jinlyang, Gyeongsan-si, \\ Kyeongsangbuk-do, Republic of Korea \\ $3^{*}$ Dept. Development, Y. H. Life Co., Ltd., B107, CU technocenter, 13-13, Hayang-ro, \\ Hayang-eup, Gyeongsan-si, Gyeongsangbuk-do, 38430, Republic of Korea \\ 1ljhcivapt@naver.com,2playeryoon@naver.com,3*dhlee8510@naver.com
}

\begin{abstract}
This study aimed to investigate the superimposition effects of extracorporeal shock wave therapy and proprioceptive neuromuscular facilitation treatment on stroke patients' spasticity reduction and improvement in upper limb function. In this study, 12 stroke patients who were under hospital treatment at a rehabilitation hospital were selected as subjects. Both the experimental and control groups underwent proprioceptive neuromuscular facilitation treatment for 30 minutes, but the experimental group received extracorporeal shock wave therapy in the upper limb for about 10 minutes before proprioceptive neuromuscular facilitation. In terms of the Fugl-Meyer assessment's wrist score, each group exhibited a statistically significant difference before and after the experiment $(P<0.05)$. In conclusion, in extracorporeal shock wave therapy and the therapeutic superimposition effect of proprioceptive neuromuscular facilitation treatment may improve hand function in stroke patients.
\end{abstract}

Keywords: Rehabilitation, Upper extremity, Function, Stroke, Spasticity

\section{Introduction}

Stroke generally causes hemiplegia in the side opposite the brain damage, thereby exhibiting impairment or disabilities. Hemiplegia is, in its most severe form, complete paralysis of half of the body. Although hemiplegic patients show a highly effective recovery of their lower limb function, their recovery of upper limb function lags far behind [1].

In addition, over $50 \%$ of patients who developed hemiplegia after a stroke experience weakened upper limb function on the affected side for a long period or permanently and are unable to use their affected upper limbs in daily living [2].

The most typical problem of stroke is hemiplegia, and most hemiplegic patients exhibit abnormal motor patterns. These abnormal patterns mainly include the persistence of primitive reflexes, flexor synergy patterns, extensor synergy patterns, abnormal muscle tension,

Article history:

Received (April 21, 2020), Review Result (May 25, 2020), Accepted (June 29, 2020) 
stiffening or hypertonicity, associated reactions, and associated movements [3]. Defects in upper limb function may be one of the most general neurological disorders that appear after a stroke.

Stroke patients' improvement in upper limb function serves as an important indicator of their functional recovery and an essential element in performing activities of daily living (ADL). Therefore, the present study aimed to investigate the effects of extracorporeal shock wave therapy on stroke patients' spasticity reduction and improvement in upper limb function.

\section{Research methods}

\subsection{Subjects}

In this study, 12 stroke patients who were under hospital treatment at a rehabilitation hospital were selected as subjects.

They were randomly divided into an experimental group $(n=6)$, who would receive extracorporeal shock wave therapy before proprioceptive neuromuscular facilitation, and a control group $(n=6)$, who would not receive extracorporeal shock wave therapy before proprioceptive neuromuscular facilitation [Figure 1].

The subjects voluntarily participated in the study after being fully informed of its purpose and contents, experimental procedures, and stability. They also submitted written participation consent before the experiment.

\subsection{Treatments}

Both the experimental and control groups underwent Proprioceptive Neuromuscular Facilitation (PNF) treatment for 30 minutes, but the experimental group received ESWT in the upper limb for about 10 minutes before proprioceptive neuromuscular facilitation. Both groups underwent their respective therapeutic interventions 18 times: three times a week for six weeks

\subsubsection{Extracorporeal shock wave therapy}

Extracorporeal shock wave therapy was applied to the myotendinous junction in the affected upper limb to reduce the upper limb's spasticity and improve its function.

For each area, the same shock wave stimulation was applied to the myotendinous junction 300 times, which translated into a total of 900 times for all areas, at a frequency of $2 \mathrm{~Hz}$ and power level of 1 .

\subsubsection{Proprioceptive neuromuscular facilitation}

Among the several types of proprioceptive neuromuscular facilitation was used as a general physical therapy for both groups in this study.

The chopping pattern is an upper limb proprioceptive neuromuscular facilitation pattern wherein the subject assumes the hook-lying position (bending the hip and knee joints in the supine position).

\subsection{Assessments}


The Korean version of the Fugl-Meyer Assessment (K-FMA) is a technique for the quantitative evaluation of stroke patients' functional recovery levels based on the phases of motor function recovery after a stroke.

Its total score is 226 points in six domains: 66 points for the upper limb, 34 points for the lower limb, 14 points for balance, 24 points for sensation, 44 points for passive joint motions, and 44 points for pain. Each item is scored0 to 2 points; a higher score indicates better motor control.

\subsection{Data analysis}

In order to verify the effectiveness of the study, the treatment method was used as an independent variable and the Fugl-Meyer assessment was used as a dependent variable.

For statistical data processing, mean \pm standard deviation values were derived from descriptive statistics using SPSS version 18.0, and an independent t-test was conducted to identify differences between the groups.

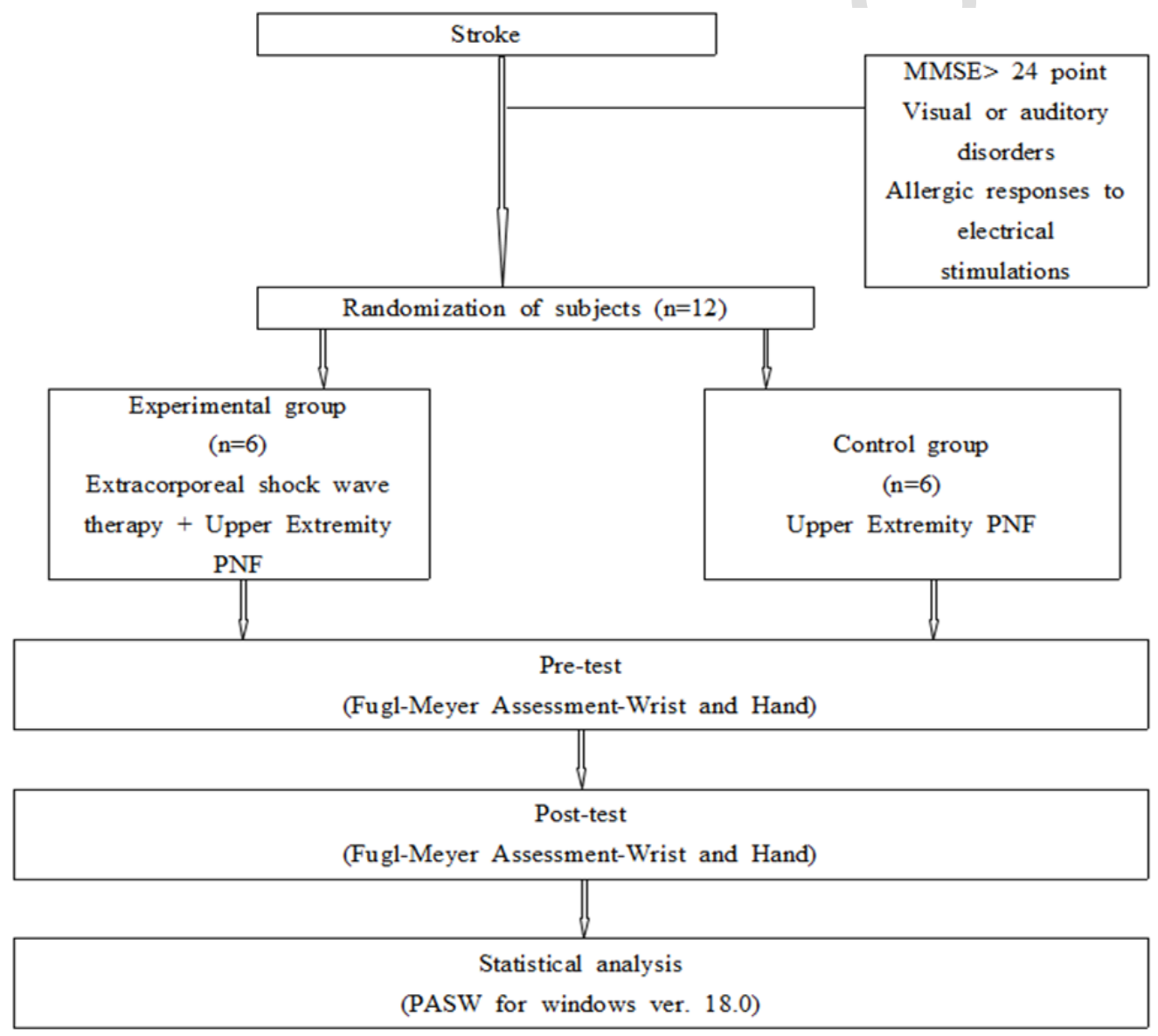

Figure 1. Flow chart

\section{Results}

In terms of the FMA's wrist score, each group exhibited a statistically significant difference before and after the experiment $(\mathrm{P}<0.05)$ [Table 1]. 
No statistically significant differences were observed between the two groups in terms of the therapeutic effects following the intervention $(\mathrm{P}>0.05)$ [Figure 2].

Table 1. Comparison of FMA score between groups

\begin{tabular}{|c|c|c|c|c|c|c|}
\hline & \multicolumn{2}{|c|}{ Experimental group } & $p$ & \multicolumn{2}{c|}{ Control group } & $p$ \\
\hline & Pre-test & Post-test & & Pre-test & Post-test & \\
\hline FMA Wrist & $4.28 \pm 1.14$ & $6.75 \pm 0.87$ & $.018^{*}$ & $4.46 \pm 1.02$ & $5.64 \pm 0.59$ & $.046^{*}$ \\
\hline pre-post & \multicolumn{2}{|c|}{$-1.96 \pm 0.23$} & & $-1.04 \pm 0.37$ & \\
\hline$p$ & \multicolumn{7}{|l|}{.064} & $6.72 \pm 1.76$ & $7.58 \pm 1.12$ & .082 \\
\hline FMA Hand & $6.89 \pm 2.02$ & $8.59 \pm 1.83$ & $.031^{*}$ & $-0.62 \pm 0.29$ & \\
\hline pre-post & $-1.44 \pm 0.36$ & & & \\
\hline$p$ & $*^{*} p<0.5$, Mean \pm SD: Mean \pm standard deviation, FMA: Fugl-Meyer Assessment \\
\hline
\end{tabular}

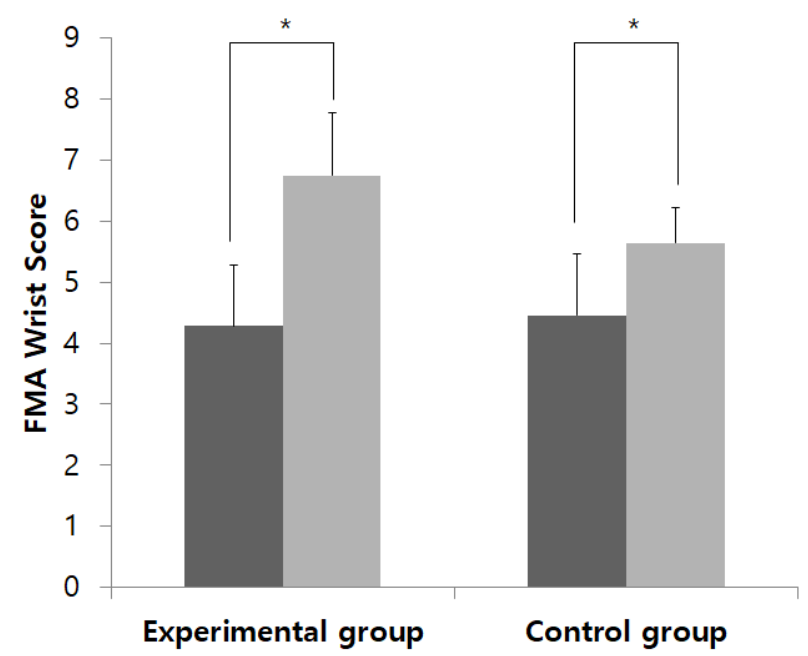

Pre-test

Post-test

${ }^{*} p<0.5$, Mean $\pm S D$ : Mean \pm standard deviation, FMA: Fugl-Meyer Assessment

Figure 2. Comparison of FMA wrist score within groups

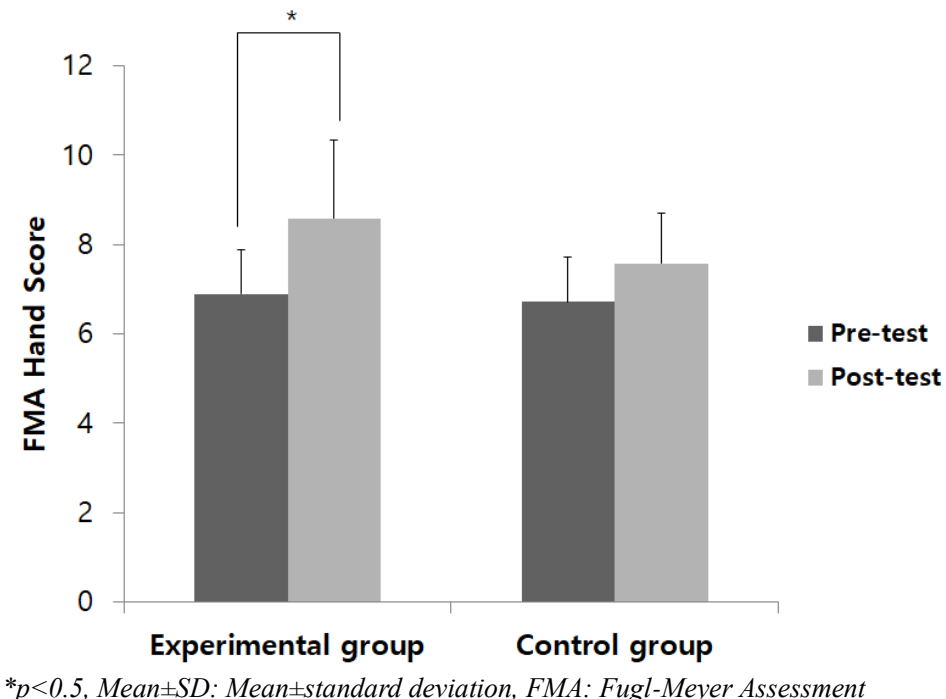

$*_{p}<0.5$, Mean \pm SD: Mean \pm standard deviation, FMA: Fugl-Meyer Assessment

Figure 3. Comparison of FMA hand score within groups 
Moreover, for the FMA's hand score, only the experimental group showed a statistically significant difference before and after the experiment $(\mathrm{P}<0.05)$ [Figure 3].

No statistically significant differences were found between the two groups in terms of their therapeutic effects following the intervention $(\mathrm{P}>0.05)$.

\section{Discussion}

Stroke is classified as obstructive stroke (in which ischemia attributed to infarction causes hypoxia and brain tissue necrosis) and hemorrhagic stroke (caused by intracranial hemorrhage). Stroke is a primary cause of death and disabilities throughout the world, and its incidence is gradually increasing [4].

Most patients develop long-term disabilities due to a chronic lack of exercise associated with a lowered quality of life. This condition is more common among the elderly, but nowadays, stroke also often occurs in those aged 20 to 40 because of changes in the social environment [5].

Particularly, because stroke patients' independence in performing ADLs depends on the functional recovery of their upper limbs, they should receive therapeutic interventions that can facilitate the upper limb's functional activity [6].

Without proper treatment, 55\% to $75 \%$ of patients exhibit upper limb disorders even six months after stroke onset [7].

Actual motor disorders that occur in a stroke patient's affected upper limb after this pathological process can be explained by the occurrence of "learned nonuse". Even a patient with a high motor recovery level at an initial evaluation learns the compensation of the unaffected upper limb in the early stages of functional training unless they spontaneously use the affected upper limb [8].

Even with the subsequent restoration of the patient's motor and sensory functions, their learned nonuse of the hand on the affected side results in motor disturbance [9].

\section{Conclusion}

This study investigated the superimposition effects of extracorporeal shock wave therapy and proprioceptive neuromuscular facilitation treatment on stroke patients' spasticity reduction and improvement in upper limb function. In the results of this study, the intervention that combined proprioceptive neuromuscular facilitation treatment with extracorporeal shock waves could not be statistically confirmed to improve the function of the hand compared to proprioceptive neuromuscular facilitation treatment alone.

However, there were many changes in hand function in the group that combined the two treatments. In the future, it is hoped that a study will be conducted to investigate the effect of the combination of various treatments on the improvement of function.

\section{Acknowledgements}

This work was supported by the National Research Foundation of Korea (NRF) grant funded by the Korea government (MSIT) (No-2019R1F1A1057731).

\section{References}

[1] E. J. Schneider, N. A. Lannin, L. Ada, and J. Schmidt, "Increasing the amount of usual rehabilitation improves activity after stroke: a systematic review," J. Physiother., vol.62, no.4, pp.182-187, (2016) DOI: 10.1016/ j.jphys.2016.08.006 
[2] K. Törnbom, H. C. Persson, J. Lundälv, and K. S., Sunnerhagen, "The impact of physical function on participation in the first year post-stroke," Acta Neurol. Scand., vol.135, no.6, pp.649-655, (2017) DOI: 10.1111/ane.12661

[3] P. Kiper, A. Szczudlik, M. Agostini, J. Opara, R. Nowobilski, L. Ventura, P. Tonin, and A. Turolla, "Virtual reality for upper limb rehabilitation in subacute and chronic stroke: a randomized controlled trial," Arch. Phys. Med. Rehabil., vol.99, no.5, pp.834-842, (2018) DOI: 10.1016/j.apmr.2018.01.023

[4] M. Morreale, P. Marchione, A. Pili, A. Lauta, S. F, Castiglia, A. Spallone, F. Pierelli, and P. Giacomini P, "Early versus delayed rehabilitation treatment in hemiplegic patients with ischemic stroke: proprioceptive or cognitive approach?” Eur. J. Phys. Rehabil. Med., vol.52, no.1, pp.81-89, (2016)

[5] M. R. El-Helow, M. L. Zamzam, M.M. Fathalla, M. A. El-Badawy, N. El Nahhas, L. M. El-Nabil, M. R. Awad, and K. Von Wild, "Efficacy of modified constraint-induced movement therapy in acute stroke," Eur. J. Phys. Rehabil. Med., vol.51, no.4, pp.371-379, (2015)

[6] B. M. A. Huisstede, L. Gebremariam, R. van der Sande, E. M. Hay, and B. W. Koes, "Evidence for effectiveness of extracorporal shock-wave therapy (ESWT) to treat calcific and non-calcific rotator cuff tendinosis--a systematic review," Man. Ther., vol.16, no.5, pp.419-433, (2011) DOI: 10.1016/j.math.2011. 02.005

[7] M. L. A. Nelson, K. A. McKellar, J. Yi, L. Kelloway, S. Munce, C. Cott, R. Hall, M. Fortin, R. Teasell, and R. Lyons, "Stroke rehabilitation evidence and comorbidity: a systematic scoping review of randomized controlled trials," Top. Stroke Rehabil., vol.24, no.5, pp.374-380, (2017) DOI: 10.1080/10749357.201 7.1282412

[8] H. Arwert, S. Schut, J. Boiten, T. Vliet Vlieland, and J. Meesters, "Patient reported outcomes of hand function three years after stroke,” Top. Stroke Rehabil., vol.25, no.1, pp.13-19, (2018) DOI: 10.1080/ 107 49357.2017.1385232

[9] J. Veldema, K. Bösl, and D. A. Nowak, "Cortico-spinal excitability and hand motor recovery in stroke: a longitudinal study,” J. Neurol., vol.265. no.5, pp.1071-1078, (2018) DOI: 10.1007/s00415-018-8802-2 